\title{
Anomalous Higgs and Triple Gauge Couplings in the Effective Field Theory Approach
}

\author{
Admir Greljo*i \\ Physik-Institut, Universität Zürich, CH-8057 Zürich, Switzerland \\ Faculty of Science, University of Sarajevo, Zmaja od Bosne 33-35, \\ 71000 Sarajevo, Bosnia and Herzegovina \\ E-mail: admirephysik.uzh.ch
}

\begin{abstract}
Given many possible forms that physics Beyond the Standard Model (BSM) could take, it is important to pursue a bottom-up approach in which as few assumptions as possible about the BSM sector are made. In this talk, we present a combined analysis of LHC Run-1 Higgs data (signal strengths) together with LEP-2 WW production measurements, in the framework of an Effective Field Theory (EFT) where the SM is extended by higher-dimensional operators suppressed by the mass scale of new physics $\Lambda$. Working under this hypothesis, important relations among Higgs and electroweak precision observables occur. We perform the first consistent analysis at the order $\Lambda^{-2}$ in the EFT expansion keeping all the relevant operators. While the two data sets suffer from flat directions, together they impose stringent model-independent constraints on the anomalous couplings, thus showing the importance of a global analysis in the EFT framework. We also discuss challenges faced when interpreting diboson production at the LHC in the EFT context.
\end{abstract}

38th International Conference on High Energy Physics

3-10 August 2016

Chicago, USA

\footnotetext{
* Speaker.

${ }^{\dagger}$ Based on the work done in collaboration with: Adam Falkowski, Martín González-Alonso, David Marzocca and Minho Son.
} 


\section{Effective field theory for the Standard Model}

The Standard Model (SM) particle content is completed with the Higgs boson discovery at the LHC Run-1. Moreover, bump searches at high $p_{T}$ failed to find new resonances, putting under pressure vanilla Beyond the Standard Model (BSM), and suggesting the scale separation between a new physics (NP) sector and the SM. Furthermore, the Higgs and the electroweak experimental program is entering precision era with the LHC Run-2. Given many possible forms BSM could take, it is important to pursue a bottom-up approach in which as few assumptions as possible about the BSM sector are made.

Effective field theory (EFT) is a suitable framework to describe infrared (IR) dynamics of an unknown ultraviolet (UV) physics. In particular, the Standard Model Effective Field Theory (SMEFT), singles out as a well-motivated framework to study BSM physics at the LHC in a model independent way. Here, the Higgs particle is the massive excitation of a pure $\mathrm{SU}(2)_{L}$ doublet. The higher-dimensional operators are constructed in terms of the doublet field $H$. The dynamical degrees of freedom are the SM fields and the SM gauge symmetry is preserved. The dominant effects are expected to rise due to the leading dimension- 6 operators:

$$
\mathscr{L}^{\mathrm{eff}}=\mathscr{L}_{\mathrm{SM}}+\sum_{i} \frac{c_{i}^{(6)}}{\Lambda^{2}} \mathscr{O}_{i}^{(6)}+\sum_{j} \frac{c_{j}^{(8)}}{\Lambda^{4}} \mathscr{O}_{j}^{(8)}+\ldots
$$

Any experimental observable (e.g. differential cross section, number of signal events in a bin, etc.), obtained from the effective Lagrangian in Eq. (1.1), takes the following form

$$
\sigma=\sigma^{\mathrm{SM}}+\sum_{i}\left(\frac{c_{i}^{(6)}}{\Lambda^{2}} \sigma_{i}^{(6 \times \mathrm{SM})}+\text { h.c. }\right)+\sum_{i j} \frac{c_{i}^{(6)} c_{j}^{(6) *}}{\Lambda^{4}} \sigma_{i j}^{(6 \times 6)}+\sum_{j}\left(\frac{c_{j}^{(8)}}{\Lambda^{4}} \sigma_{j}^{(8 \times \mathrm{SM})}+\text { h.c. }\right)+\ldots
$$

It is important to notice that the $D=6$ squared terms are of the same order in the EFT expansion parameter $\Lambda$ as the (neglected) interference of the $D=8$ with the SM. Working consistently at order $\mathscr{O}\left(\Lambda^{-2}\right)$ means keeping only linear dependence on the Wilson coefficients (that is, dropping the $D=6$ squared terms) in the physical observables. In other words, $D=6$ squared terms formally represent partial next-to-leading order corrections. A good practice would be to check whether or not including these terms impacts the numerical results significantly, which would give an idea about the convergence of the series. Note also that, since different bases of $D=6$ operators in the literature differ by $\mathscr{O}\left(\Lambda^{-4}\right)$ terms corresponding to $D>6$ operators, only results obtained consistently at $\mathscr{O}\left(\Lambda^{-2}\right)$ are basis-independent.

\section{Global fit to the LHC Higgs and LEP-2 WW data}

In the presence of $D=6 \mathrm{CP}$-conserving operators, the cubic couplings of electroweak gauge bosons take the form:

$$
\begin{aligned}
\mathscr{L}_{\mathrm{tgc}} & =i e\left(W_{\mu \nu}^{+} W_{\mu}^{-}-W_{\mu \nu}^{-} W_{\mu}^{+}\right) A_{v}+i e \frac{c_{\theta}}{s_{\theta}}\left(1+\delta g_{1, z}\right)\left(W_{\mu \nu}^{+} W_{\mu}^{-}-W_{\mu \nu}^{-} W_{\mu}^{+}\right) Z_{v} \\
& +i e\left(1+\delta \kappa_{\gamma}\right) A_{\mu \nu} W_{\mu}^{+} W_{v}^{-}+i e \frac{c_{\theta}}{s_{\theta}}\left(1+\delta \kappa_{z}\right) Z_{\mu \nu} W_{\mu}^{+} W_{v}^{-} \\
& +i \frac{\lambda_{z} e}{m_{W}^{2}}\left[W_{\mu \nu}^{+} W_{v \rho}^{-} A_{\rho \mu}+\frac{c_{\theta}}{s_{\theta}} W_{\mu \nu}^{+} W_{v \rho}^{-} Z_{\rho \mu}\right],
\end{aligned}
$$


where $\delta \kappa_{z}=\delta g_{1, z}-\frac{s_{\theta}^{2}}{c_{\theta}^{2}} \delta \kappa_{\gamma}, s_{\theta}$ is the sine of the Weinberg angle, and $c_{\theta}=\sqrt{1-s_{\theta}^{2}}$. Therefore, as long as operators with $D>6$ are negligible, deformations of the cubic gauge interactions due to NP can be parametrized by three anomalous Triple Gauge Couplings (aTGC): $\delta g_{1, z}, \delta \kappa_{\gamma}$, and $\lambda_{z}$. In the SM limit, $\delta g_{1, z}=\delta \kappa_{\gamma}=\lambda_{z}=0$. Non-zero aTGC are effectively generated in models with new heavy particles, after the latter are integrated out at low energies.

In Ref. [1], we derive constraints on the aTGC and anomalous Higgs couplings from the combined LHC Higgs and LEP-2 WW data sets. In our analysis, all $D=6$ operators affecting Higgs couplings to matter and gauge boson self-couplings are allowed to be simultaneously present with arbitrary coefficients, assuming minimal flavor violation (MFV). In the Higgs basis, these parameters are [2]:

$$
\delta c_{z}, c_{z z}, c_{z \square}, c_{\gamma \gamma}, c_{z \gamma}, c_{g g}, \delta y_{u}, \delta y_{d}, \delta y_{e}, \lambda_{z} .
$$

The relation of these parameters to the interaction terms in the Lagrangian, as well as the relation to the aTGC, can be found in Ref. [2]. As discussed before, we only take into account linear corrections in the Wilson coefficients, thus working consistently at the $\mathscr{O}\left(\Lambda^{-2}\right)$ in the EFT expansion.

For the LEP-2 WW data, we use the measured total and differential $e^{+} e^{-} \rightarrow W^{+} W^{-}$cross sections at different collider energies. Given the stringent electroweak precision (EWP) constraints on the $\mathrm{Z}$ and $\mathrm{W}$ propagators and couplings to electrons from LEP-1, these measurements can effectively constrain three linear combinations of Wilson coefficients of $D=6$ operators that correspond to the aTGC. We use this dependence to construct the likelihood function $\chi_{W W}^{2}\left(\delta g_{1, z}, \delta \kappa_{\gamma}, \lambda_{z}\right)$. For the LHC Higgs data, we use the signal strength observables, that is, the ratios between the measured Higgs yield and its SM prediction $\mu \equiv(\sigma \times \mathrm{BR}) /(\sigma \times \mathrm{BR}) \mathrm{SM}$. All the measurements used in the analysis are summarized in Ref. [1]. The effect of $D=6$ operators on the signal strengths was calculated for each channel and production mode. After imposing EWP constraints, nine linear combinations of $D=6$ operators can affect signal strengths in an observable way. The crucial point is that two of these combinations correspond to the aTGC $\delta g_{1, z}$, and $\delta \kappa_{\gamma}$. Therefore, the likelihood function constructed from the LHC Higgs data, $\chi_{h}^{2}\left(\delta g_{1, z}, \delta \kappa_{\gamma}, \ldots\right)$, may lead to additional constraints on aTGC.

Indeed, combining the two likelihoods, $\chi_{\text {comb. }}^{2}=\chi_{h}^{2}+\chi_{W W}^{2}$, we obtain strong constraints on the aTGC at the level of $\mathscr{O}(0.1)$. We find the following central values, $1 \sigma$ errors, and the correlation matrix for the aTGC:

$$
\left(\begin{array}{c}
\delta g_{1, z} \\
\delta \kappa_{\gamma} \\
\lambda_{z}
\end{array}\right)=\left(\begin{array}{c}
0.043 \pm 0.031 \\
0.142 \pm 0.085 \\
-0.162 \pm 0.073
\end{array}\right), \quad \rho=\left(\begin{array}{ccc}
1 & 0.74 & -0.85 \\
0.74 & 1 & -0.88 \\
-0.85 & -0.88 & 1
\end{array}\right),
$$

after minimizing the combined likelihood with respect to the remaining seven Wilson coefficients. These constraints hold in any new physics scenario predicting approximately flavor blind coefficients of $D=6$ operators and in which $D>6$ operators are subleading. The constraints on all ten Wilson coefficients entering the analysis are reported in the supplemental material of Ref. [2]. They are given in different bases for reader's convenience together with the full correlation matrices.

Let us discuss here qualitatively the most important features of our numerical results. Higgs data are sensitive to $\delta g_{1, z}$ and $\delta \kappa_{\gamma}$ primarily via their contribution to electroweak Higgs production channels. However, only one combination of the two aTGC is strongly constrained, while the 


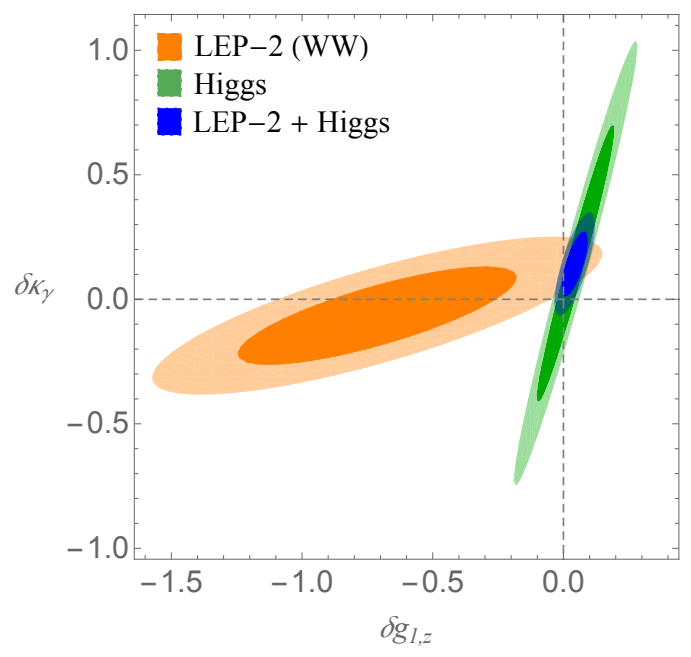

Figure 1: Allowed 68\% and 95\% CL region in the $\left(\delta g_{1, z}, \delta \kappa_{\gamma}\right)$ plane after considering LEP-2 $W W$ production data (aTGC), LHC Run-1 Higgs signal strengths, and the combination of both data sets (see Ref. [1]).

bound on the direction $\delta \kappa_{\gamma} \approx 3.8 \delta g_{1, z}$ is very weak. Analogously, also LEP-2 WW bounds present an approximate blind direction. This is illustrated in Fig. 1, where the WW and Higgs constraints in the $\delta g_{1, z}-\delta \kappa_{\gamma}$ plane are shown separately. Since the flat directions are nearly orthogonal, combining LHC Higgs and LEP-2 WW data leads to the non-trivial constraints on aTGC, advocating the importance of a global approach in the EFT studies.

\section{Anomalous Triple Gauge Couplings at the LHC}

Diboson production processes $\left(p p \rightarrow W^{+} W^{-}\right.$or $W^{ \pm} Z$ ) at Tevatron and LHC are also very sensitive to aTGC. However, these measurements were not included in the previous global analysis of Ref. [1], because their EFT interpretation is much more involved [3]. The main complication comes from the fact that hadron collisions probe a wide range of energies, part of which may be beyond the validity regime of the EFT approach. This is in contrast with LEP-2 observables and on-shell Higgs decay measurements, where the typical energy scale is the LEP collider energy and Higgs mass, respectively. Formally, the EFT expansion is more slowly convergent due to the large $\hat{s} / \Lambda^{2}$ factor. This enhances the sensitivity to neglected $D=8$ operators and complicates the extraction of robust aTGC bounds from LHC data. The situation is further worsened since the linear effects of $D=6$ operators (coming from its interference with the SM) happen to be suppressed in these observables [3]. Indeed, we find that including consistently at $\mathscr{O}\left(\Lambda^{-2}\right)$ these LHC measurements in the global analysis, has negligible effects on the results of Ref. [1].

Nevertheless, it is important to note that in a wide class of BSM models with some strongly coupled sector, the contribution from $D=8$ operators is subleading with respect to $D=6$ squared terms. This can be understood from a simple matching of the Wilson coefficients to the UV parameters of the theory: $c_{i}^{(6)} \sim c_{j}^{(8)} \sim g_{*}^{2}$, where $g_{*}^{2} \gg 1$ is a strong coupling. This implies that the $D=6$ squared terms dominate over the linear $D=8$ by a factor $g_{*}^{2} / g_{\text {SM }}^{2} \gg 1$. Consequently, present aTGC extractions from the LHC data are justified for these BSM scenarios. Even in such cases it is convenient to perform the EFT analysis using different cuts on the appropriate kinemati- 
cal variables [3]. Ideally, if the full invariant mass of the $V V$ system (or equivalently $\sqrt{\hat{s}}$ ) could be reconstructed from data, one would impose an appropriate cut on $m_{V V}$ on both data and simulated events, allowing to build the likelihood using expected and observed cross sections with the cuts, i.e.

$$
\left(\sigma_{\mathrm{SM}}+\sigma_{\mathrm{BSM}}\right)\left(m_{V V}<m_{V V}^{\max }\right), \quad \sigma_{\mathrm{obs}}\left(m_{V V}<m_{V V}^{\max }\right) .
$$

In this way one would derive bounds consistently, with the EFT applicable to theories in which new states are heavier than $m_{V V}^{\max }$.

However, in realistic analyses this approach is limited by the incapability of reconstructing the full invariant mass of the diboson system when one or both gauge bosons decay into neutrinos $(p p \rightarrow W W \rightarrow \ell \ell v v)$. In this case other observables, which we generically denote as $M_{v i s}$, are constructed from the available information in the final state. For example, these can be the dilepton invariant mass $m_{\ell \ell}$ in the case of $W W$, the transverse mass $m_{T}^{W Z}$ in the case of $W Z$ production, or the transverse momentum of a gauge boson $p_{T}(V)$. The problem with this approach is that all these observables exhibit a poor correlation with the physically relevant scale $m_{V V}$ [3].

In this situation one can still set conservative bounds on the EFT parameters, imposing the EFT cut $m_{V V}^{\max }$ only on the simulated BSM events (not on the SM) and comparing with the observed events. A simple way to understand this approach is to simplify the $\chi^{2}$ analysis by approximating that the $68 \%$ CL bound comes from comparing the measured cross section in a given bin of the experimentally accessible distribution, $\sigma_{o b s} \pm \Delta \sigma$, with the expected one, $\sigma_{\mathrm{SM}}+\sigma_{\mathrm{BSM}}$, and requiring the latter to be within the experimental error, namely

$$
\sigma_{o b s}-\Delta \sigma<\sigma_{\mathrm{SM}}+\sigma_{\mathrm{BSM}}<\sigma_{o b s}+\Delta \sigma
$$

By applying the $m_{V V}$ cut on the BSM events, at the simulation level, we split $\sigma_{\mathrm{BSM}}=\sigma_{\mathrm{BSM}}^{m_{V V}<m_{V V}^{\max }}+$ $\sigma_{\mathrm{BSM}}^{m_{V V}>m_{V V}^{\max }}$. If both these terms are positive and as long as no significant excess is observed, then from the inequalities in eq. (3.2) follows

$$
\sigma_{o b s}-\sigma_{\mathrm{SM}}-\Delta \sigma<\sigma_{\mathrm{BSM}}^{m_{V V}<m_{V V}^{\max }}<\sigma_{o b s}-\sigma_{\mathrm{SM}}+\Delta \sigma .
$$

Under the above-mentioned assumptions, the resulting constraint on $\sigma_{\mathrm{BSM}}^{m_{V V}<m_{V V}^{\max }}$ provides a conservative bound on the EFT parameters, with the first inequality trivially satisfied.

Note that the positivity assumption is not necessarily realized in general. The BSM contributions are schematically given by

$$
\sigma_{\mathrm{BSM}} \propto\left(\mathscr{A}_{\mathrm{SM}}^{*} \mathscr{A}_{\mathrm{BSM}}+\text { h.c. }\right)+\left|\mathscr{A}_{B S M}\right|^{2}
$$

and can be negative if the interference terms dominates and is negative. However, as discussed before, in the parameter space where the BSM effects are large enough to be observable, the quadratic terms typically dominate the low-energy part of the cross section where the EFT approach is reliable. Assuming also $D=8$ contribution to be sub-leading implies $\sigma_{\mathrm{BSM}}^{m_{V V}<m_{V V}^{\max }}$ is positive. Furthermore, for large invariant masses (where the EFT is no longer valid) one would naively expect that the interference effect in this region is generally small due to a relatively small $\mathscr{A}_{\mathrm{SM}}$, which may justify assuming a positive $\sigma_{\mathrm{BSM}}^{m_{V V}>m_{V V}^{\max }}$.

We use this method to recast a selection of ATLAS and CMS $W W$ and $W Z$ analyses, both with $8 \mathrm{TeV}$ and $13 \mathrm{TeV}$ data, in order to extract consistent bounds on aTGC in the EFT [3]. The limits as a function of the cut $m_{V V}^{\max }$ are shown in Fig. 2, with more details provided in Ref. [3]. 

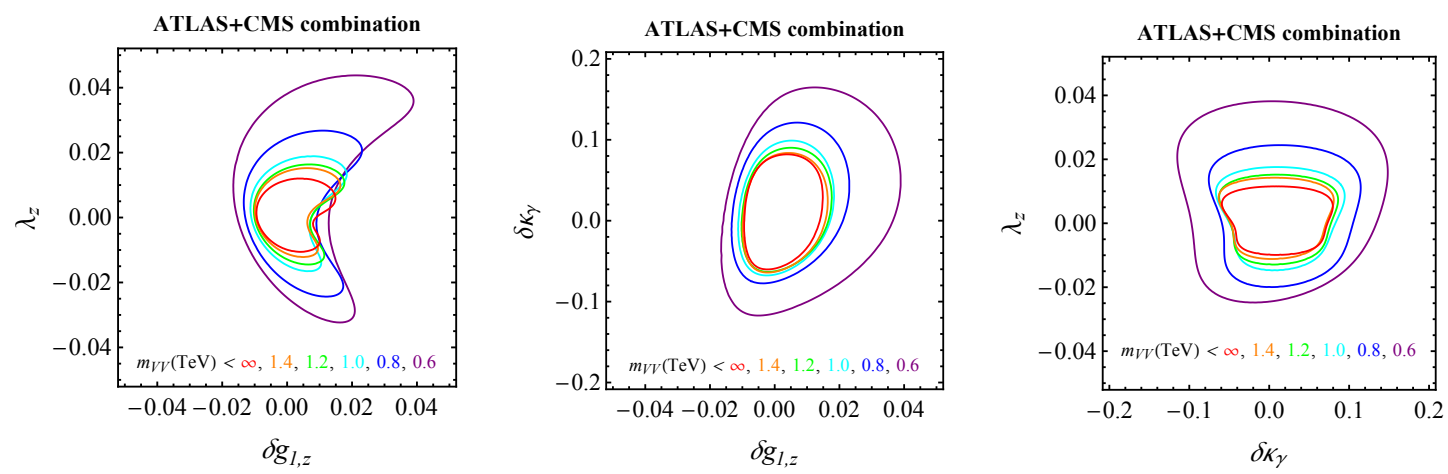

Figure 2: Combined 68\% CL region from CMS $W W(8 \mathrm{TeV})$ and ATLAS $W Z(8+13 \mathrm{TeV})$ searches for different $m_{V V}$ cuts from Ref. [3].

\section{Conclusions}

To sum up, by working at $\mathscr{O}\left(\Lambda^{-2}\right)$ in the EFT and under the MFV assumption, in Ref. [1] we obtained strong and model-independent bounds on the aTGC and anomalous Higgs couplings via the combination of LEP-2 WW and LHC Higgs signal-strength data. Interestingly enough, the combination of the two data sets lifts the flat direction present in each of them taken separately. As a result, the bounds do not change significantly when the (formally subleading) $D=6$ squared contributions are included in the analysis. Thus, these results are robust and model-independent. They can be easily translated to any given BSM model (that can be matched to the SMEFT) without having to redo the analysis of the data.

On the other hand, diboson production at the LHC does not improve the global fit result [3]. To control the validity of the EFT in LHC searches one should impose a cut on high- $p_{T}$ events, $m_{V V}^{\max }<\Lambda$, where $\Lambda$ is the assumed mass scale of the new physics, and perform the analysis for different values of $m_{V V}^{\max }$ (i.e. different assumptions on $\Lambda$ ). In this way, the EFT interpretation of the bounds for theories with a lower cut-off could also be possible. However, a complication arises due to the fact that the kinematical variable $m_{V V}$ is very badly correlated with the measured variables, implying that a cut on the latter does not remove the unwanted high- $p_{T}$ events with a good enough efficiency. In this case, by imposing the desired $m_{V V}^{\max }$ cut at the simulation level on the BSM events only, consistent and conservative EFT bounds can still be obtained if no significant excess from the SM is observed [3].

\section{References}

[1] A. Falkowski, M. Gonzalez-Alonso, A. Greljo and D. Marzocca, Phys. Rev. Lett. 116, no. 1, 011801 (2016) doi:10.1103/PhysRevLett.116.011801 [arXiv:1508.00581 [hep-ph]].

[2] A. Falkowski, Pramana 87, no. 3, 39 (2016) doi:10.1007/s12043-016-1251-5 [arXiv:1505.00046 [hep-ph]].

[3] A. Falkowski, M. Gonzalez-Alonso, A. Greljo, D. Marzocca and M. Son, arXiv:1609.06312 [hep-ph]. 